\title{
Householder triangularization of a quasimatrix
}

\author{
LLOYD N. TREFETHEN \\ Oxford Computing Laboratory, Wolfson Bldg., Parks Rd., \\ Oxford $O X 13 Q D$, $U K$.
}

[Received on 4 July 2008]

\begin{abstract}
A standard algorithm for computing the $\mathrm{QR}$ factorization of a matrix $A$ is Householder triangularization. Here this idea is generalized to the situation in which $A$ is a quasimatrix, that is, a "matrix" whose "columns" are functions defined on an interval $[a, b]$. Applications are mentioned to quasimatrix leastsquares fitting, singular value decomposition, and determination of ranks, norms, and condition numbers, and numerical illustrations are presented using the chebfun system.
\end{abstract}

Keywords: Householder triangularization, QR factorization, chebfun, quasimatrix, SVD

\section{QR factorization and Householder triangularization}

Let $A$ be an $m \times n$ matrix, $m>n$. A (reduced) QR factorization of $A$ is a factorization

$$
A=Q R, \quad\left[\begin{array}{llll}
\mathrm{x} & \mathrm{x} & \mathrm{x} & \mathrm{x} \\
\mathrm{x} & \mathrm{x} & \mathrm{x} & \mathrm{x} \\
\mathrm{x} & \mathrm{x} & \mathrm{x} & \mathrm{x} \\
\mathrm{x} & \mathrm{x} & \mathrm{x} & \mathrm{x} \\
\mathrm{x} & \mathrm{x} & \mathrm{x} & \mathrm{x} \\
\mathrm{x} & \mathrm{x} & \mathrm{x} & \mathrm{x}
\end{array}\right]=\left[\begin{array}{llll}
\mathrm{q} & \mathrm{q} & \mathrm{q} & \mathrm{q} \\
\mathrm{q} & \mathrm{q} & \mathrm{q} & \mathrm{q} \\
\mathrm{q} & \mathrm{q} & \mathrm{q} & \mathrm{q} \\
\mathrm{q} & \mathrm{q} & \mathrm{q} & \mathrm{q} \\
\mathrm{q} & \mathrm{q} & \mathrm{q} & \mathrm{q} \\
\mathrm{q} & \mathrm{q} & \mathrm{q} & \mathrm{q}
\end{array}\right]\left[\begin{array}{llll}
\mathrm{r} & \mathrm{r} & \mathrm{r} & \mathrm{r} \\
& \mathrm{r} & \mathrm{r} & \mathrm{r} \\
& & \mathrm{r} & \mathrm{r} \\
& & \mathrm{r}
\end{array}\right.
$$

where $Q$ is $m \times n$ with orthonormal columns and $R$ is upper-triangular. Here and in subsequent equations we illustrate our matrix operations by schematic pictures in which $x$ denotes an arbitrary entry, $r$ denotes an entry of an upper-triangular matrix, $q$ denotes an entry of an orthonormal column, and a blank denotes a zero. QR factorization is a fundamental step for all kinds of computations in numerical linear algebra, including least-squares, eigenvalues, and singular value decomposition (SVD) (Björck (1996); Golub \& Van Loan (1996); Stewart (1998b); Trefethen \& Bau (1997)).

One way to compute a QR factorization is by Gram-Schmidt or modified Gram-Schmidt factorization, a process of triangular orthogonalization. This is fast and straightforward, but the calculation may break down with division by zero if $A$ is rank-deficient, that is, if its column space has dimension $<n$. Even if $A$ has full rank, the matrix $Q$ computed in floating point arithmetic may be far from orthogonal if $A$ is ill-conditioned, and this may have an adverse effect on the accuracy of subsequent computations (Stewart (1998b)).

In 1958 Alston Householder introduced an alternative approach to (1.1) based on orthogonal triangularization, which has become one of the standard tools of computational science (Householder (1958)). At the first step, a unitary matrix $H_{1}$ is applied that introduces zeros below the diagonal in the first column:

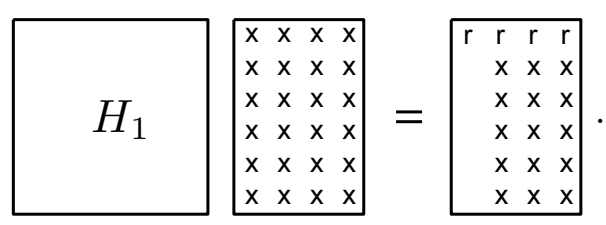


The second step applies another unitary matrix $\mathrm{H}_{2}$ that leaves the first row unchanged and introduces zeros below the diagonal in the second column:

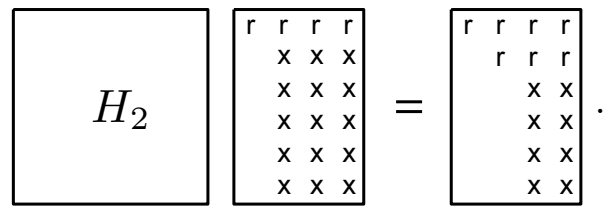

After $n$ steps, $A$ has become upper-triangular:

$$
H_{n} \cdots H_{2} H_{1} A=T \cdot \quad H \quad\left[\begin{array}{llll}
\mathrm{x} & \mathrm{x} & \mathrm{x} & \mathrm{x} \\
\mathrm{x} & \mathrm{x} & \mathrm{x} & \mathrm{x} \\
\mathrm{x} & \mathrm{x} & \mathrm{x} & \mathrm{x} \\
\mathrm{x} & \mathrm{x} & \mathrm{x} & \mathrm{x} \\
\mathrm{x} & \mathrm{x} & \mathrm{x} & \mathrm{x} \\
\mathrm{x} & \mathrm{x} & \mathrm{x} & \mathrm{x}
\end{array}\right]=\left[\begin{array}{llll}
\mathrm{r} & \mathrm{r} & \mathrm{r} & \mathrm{r} \\
\mathrm{r} & \mathrm{r} & \mathrm{r} \\
& \mathrm{r} & \mathrm{r} \\
& \mathrm{r} \\
& \\
\end{array}\right.
$$

Multiplying on the left by $H^{*}=H_{1} H_{2} \ldots H_{n}$ now gives

$$
A=H_{1} H_{2} \cdots H_{n} T \quad\left[\begin{array}{llll}
x & x & x & x \\
x & x & x & x \\
x & x & x & x \\
x & x & x & x \\
x & x & x & x \\
x & x & x & x
\end{array}\right]=\left[\begin{array}{llll}
r & r & r & r \\
r & r & r \\
& r & r \\
& r \\
& & r
\end{array}\right.
$$

We have written $H_{k}$ instead of $H_{k}^{-1}$, for the two are the same since the Householder matrices $H_{k}$ are self-adjoint as well as unitary. In fact, $H_{k}$ is a reflection matrix, which maps $\mathbb{C}^{n}$ to itself by reflection across an $(n-1)$-dimensional hyperplane. This interpretation makes it clear that $H_{k}^{2}=I$, i.e., $H_{k}^{-1}=H_{k}$. Algebraically $H_{k}$ takes the form

$$
H_{k}=I-2 v_{k} v_{k}^{*}
$$

where $v_{k}$ is a unit vector, i.e., $\left\|v_{k}\right\|=1$. (Throughout this paper, ${ }^{*}$ is the conjugate transpose and $\|\cdot\|$ is the 2-norm.) To multiply a Householder reflection by a vector or matrix, one always uses this form rather than an explicit entrywise representation, and that is why our schematic figures show $H$ and $H^{*}$ as boxes with no entries inside them.

For any unit vector $v_{k},(1.4)$ defines a reflection in the sense just defined. In the application to QR factorization, $v_{k}$ is chosen orthogonal to the unit vectors $e_{1}, \ldots, e_{k-1}$, i.e., with zeros in positions $1, \ldots, k-1$, and this choice ensures that the space spanned by these vectors lies in the hyperplane that is invariant under $H_{k}$, which in turn ensures that zeros introduced at one step are not destroyed at later steps.

The factorization (1.3) is almost (1.1), but we are not quite there yet since the matrices $H^{*}$ and $T$ on the right have dimensions $m \times m$ and $m \times n$ rather than $m \times n$ and $n \times n$. To achieve the latter we need to delete all but the first $n$ columns of $H^{*}$ and all but the first $n$ rows of $T$, which will have no effect on the product since the deleted rows are all zero. But if $H^{*}=H_{1} \cdots H_{n}$ is never actually formed as a matrix, how do we delete some of its columns? We give an answer that will make the transition to quasimatrices particularly convenient as well as clarifying the treatment of matrices with complex entries. We shall factor $T$ in (1.2) into a product of three matrices: an $m \times n$ "rectangular identity" $E$, whose columns are the unit vectors $e_{1}, \ldots, e_{n}$; an $n \times n$ "sign matrix" $S=\operatorname{diag}\left(s_{1}, \ldots, s_{n}\right)$, whose diagonal entries are real or 
complex numbers with $\left|s_{j}\right|=1$; and an $n \times n$ upper-triangular matrix $R$ whose diagonal entries are real and nonnegative:

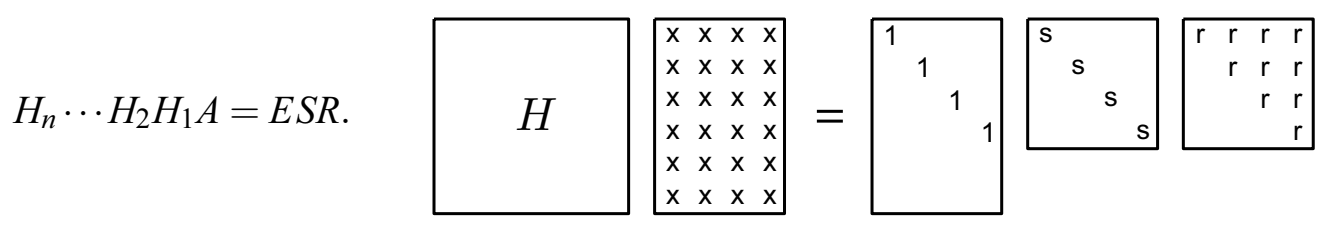

This formulation makes it clear that we can compute the $m \times n$ matrix $Q$ by applying $H_{n}, \ldots, H_{1}$ successively to $E S$ :

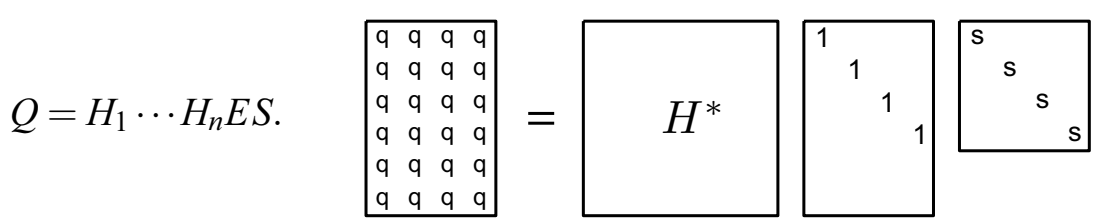

\section{Generalization to quasimatrices}

Our subject in this note is the generalization of these ideas to the situation in which $A$ is not a matrix but a quasimatrix defined on an interval $[a, b]$. By this we mean an " $\infty \times n$ matrix" whose "columns" are functions of $x \in[a, b]$. Thus $A$ represents a linear map from $\mathbb{C}^{n}$ to $L^{2}[a, b]$. The term quasimatrix comes from Stewart (1998), and the same idea was introduced with different terminology in de Boor (1991) and (Trefethen \& Bau, 1997, p. 52). We assume that each column lies in $L^{2}[a, b]$, so that inner products make sense, which we shall write in vector notation $v^{*} w ;\|\cdot\|$ is now the $L^{2}$ norm on $[a, b]$. For example one might consider the quasimatrix with columns corresponding to the functions $1, x, x^{2}, \ldots, x^{n-1}$ defined on $[-1,1]$. The columns of $Q$ in its QR factorization will be multiples of the Legendre polynomials $P_{0}, \ldots, P_{n-1}$, and the first few entries of $R$ are $r_{11}=2^{1 / 2}, r_{12}=0, r_{22}=(2 / 3)^{1 / 2}, r_{13}=(2 / 9)^{1 / 2}$, $r_{23}=0, r_{33}=(8 / 45)^{1 / 2}$.

If $A$ is a quasimatrix, the QR factorization formula (1.1) still makes sense. Now $Q$, like $A$, has dimensions $\infty \times n$, and it has orthonormal columns:

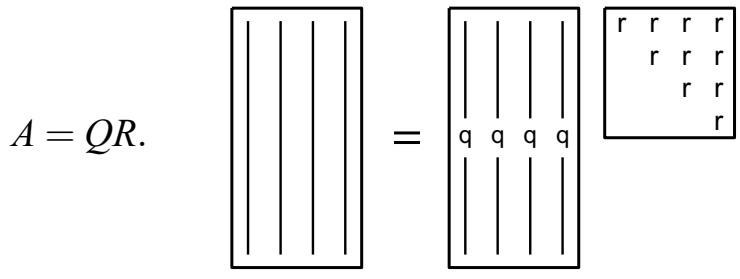

A Gram-Schmidt computation goes exactly as before, with discrete inner products replaced by continuous ones, and is susceptible to the same difficulties if $A$ is ill-conditioned or rank-deficient. GramSchmidt orthogonalization of quasimatrices was discussed in Battles \& Trefethen (2004) and Battles (2006).

Another way to compute the $\mathrm{QR}$ decomposition of $A$ would be to form $A^{*} A$, which is an $n \times n$ matrix, and then find its Cholesky factorization $A^{*} A=R^{*} R$ by standard methods. One then has $Q=A R^{-1}$, at least if $A$ has full rank so that $R$ is nonsingular. Like Gram-Schmidt orthogonalization, this method has difficulties if $A$ is ill-conditioned, since it "squares the condition number." On the other hand it provides 
an easy proof of a basic proposition: if the quasimatrix $A$ has rank $n$ and $R$ has positive diagonal entries, then the QR factorization is unique.

Our aim is to compute (2.1) by the numerically more stable method of Householder reflections, which has apparently not been done before. The very first step introduces the crucial question. What could it mean to map a function onto another function that is "zero below the diagonal"? The answer we propose is that one should start from a quasimatrix analogue of (1.5):

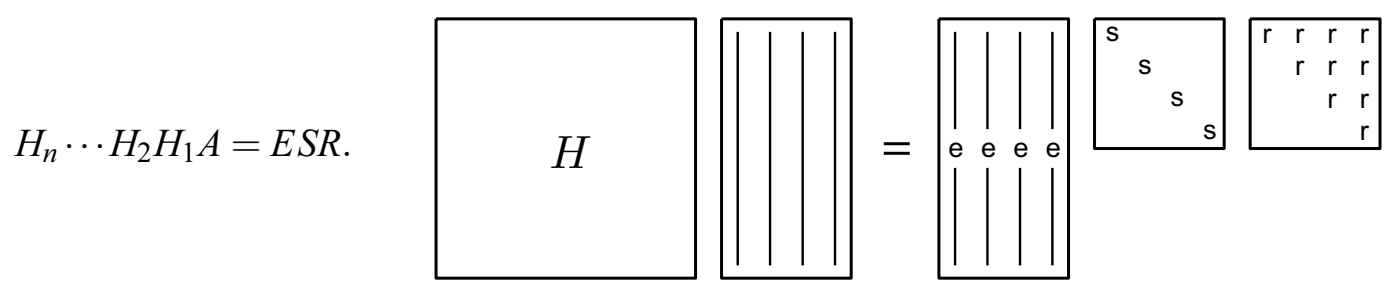

Here $E$ will be an $\infty \times n$ quasimatrix, fixed in advance, with orthonormal columns $\left\{e_{j}\right\}$, and $S$ will be an $n \times n$ sign matrix as before. In practice we take $e_{j}$ to be a multiple of the $(j-1)$ st Legendre polynomial $P_{j-1}(x)$, scaled to $[a, b]$. The target of the Householder reflector $H_{k}$ will be not a vector zero below position $k$, but a function in the space spanned by $e_{1}, \ldots, e_{k}$. Again the signs $\left\{s_{j}\right\}$ will be chosen so that the diagonal entries of $R$ are real and nonnegative.

The first step of Householder quasimatrix triangularization looks like this:

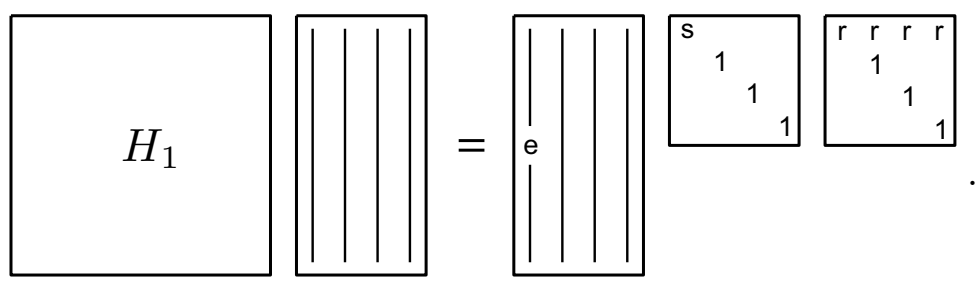

A Householder reflector $H_{1}$ has been applied to $A$, changing all its columns and in particular changing the first column to $r_{11} s_{1} e_{1}$. The second step looks like this:

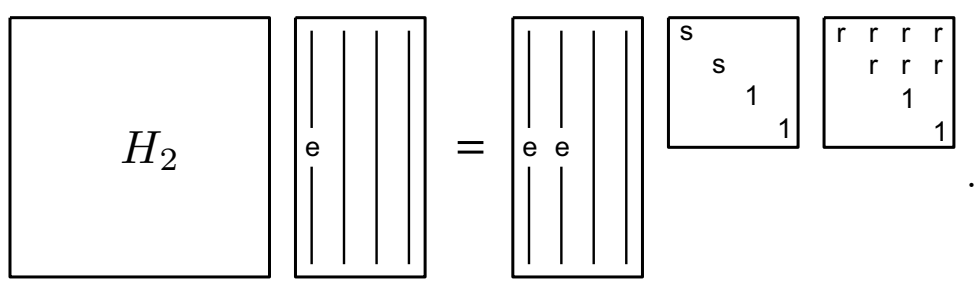

The reflector $H_{2}$ has now changed columns 2 through $n$, converting column 2 to $r_{22} s_{2} e_{2}$. The process continues in this fashion until the form (2.2) is achieved at step $n$.

The precise formulas for these computations are as follows, for each step $k$ from 1 to $n$ :

$$
\begin{gathered}
x=A(:, k), r_{k k}=\|x\|, \quad s_{k}=-\operatorname{sign}\left(e_{k}^{*} x\right), \quad y=s_{k} r_{k k} e_{k}, \quad v=\frac{y-x}{\|y-x\|}, H_{k}=I-2 v v^{*}, \\
r_{k j}=e_{k}^{*} A(:, j), \quad A(:, j)=A(:, j)-r_{k j} e_{k}, \quad k+1 \leqslant j \leqslant n .
\end{gathered}
$$

(The function $\operatorname{sign}(z)$ returns $z /\|z\|$ if $z \neq 0,1$ if $z=0$.) The operations of (2.4) subtract off projections of the current vector $e_{k}$ from the remaining columns of $A$. 
After triangularization is completed, the computation of $Q$ if it is needed goes as in (1.6):

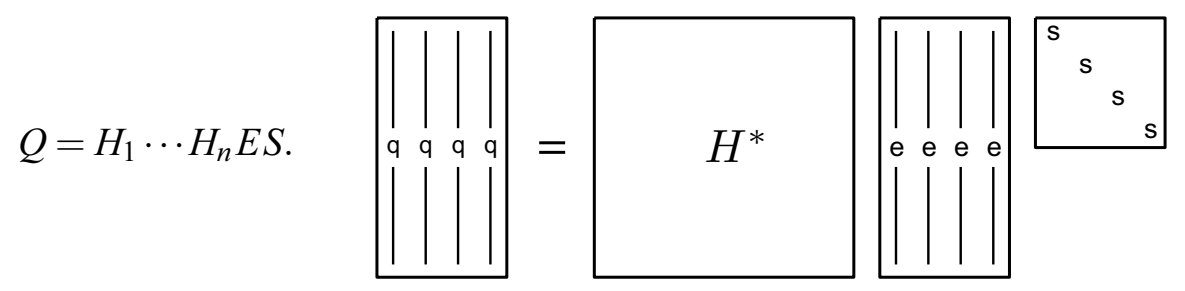

Here is our algorithm written in MATLAB-style pseudocode. The input is an $\infty \times n$ quasimatrix $A$, and the output is an $\infty \times n$ orthonormal quasimatrix $Q$ and an $n \times n$ upper-triangular matrix $R$ with nonnegative diagonal entries satisfying (2.1). One difference from the description above is that, instead of accumulating the signs $s_{k}$, we replace $e_{k}$ by $s_{k} e_{k}$ at step $k$ before moving on to step $k+1$. Another is that an additional line has been added to "improve orthogonality" of the direction vector $v_{k}$ to the previous target vectors $e_{1}, \ldots, e_{k-1}$. In exact arithmetic this would not be needed, but in floating point arithmetic it makes a significant difference.

\section{HOUSEHOLDER TRIANGULARIZATION OF AN $\infty \times n$ QUASIMATRIX A}

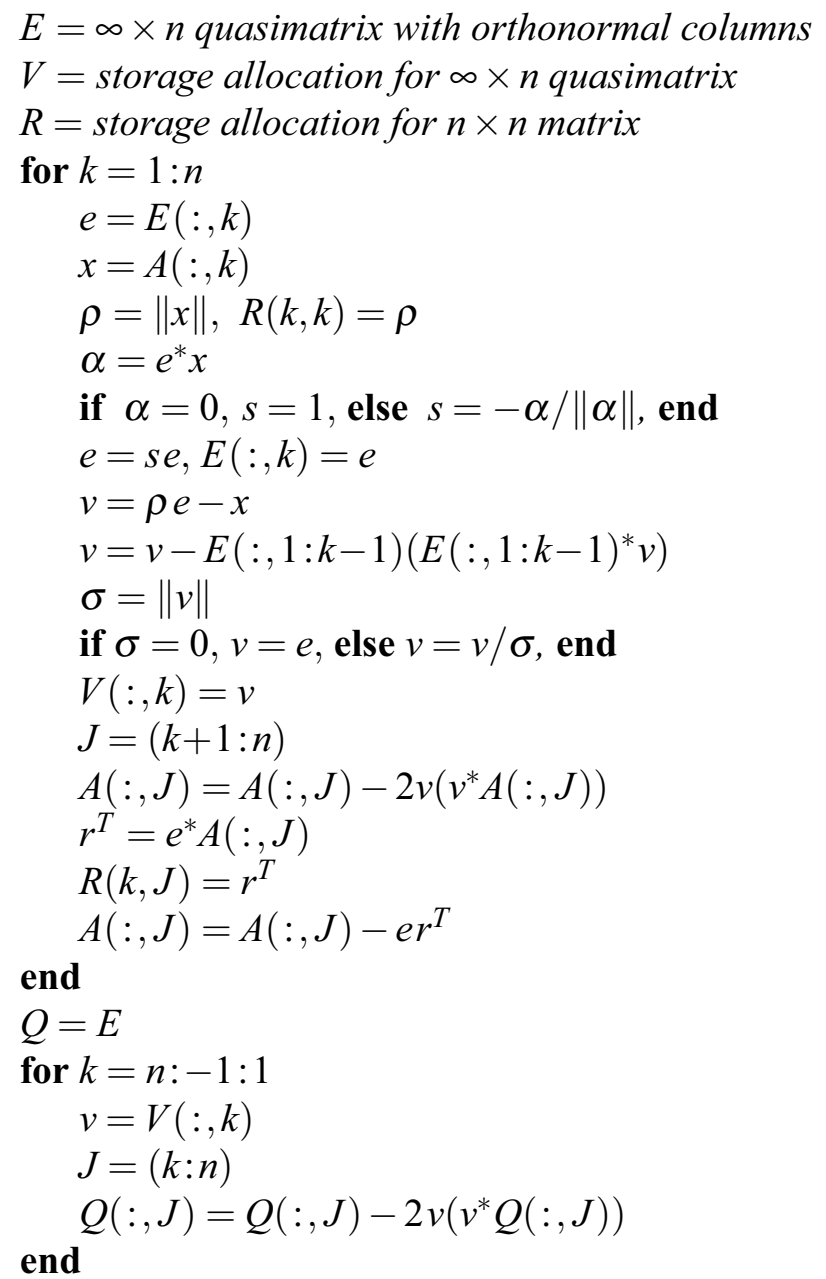

TRIANGULARIZATION

target for this reflection

vector to be mapped to $e$

compute real or complex sign

modify $e$ to take account of sign

vector defining reflection

improve orthogonality

If zero column, arbitrary reflection

store Householder vector

convenient abbreviation

apply reflection

entries of row $k$ of $R$

FORMATION OF $Q$

retrieve Householder vector

convenient abbreviation

apply reflection 


\section{Least-squares and pseudoinverse}

The most basic application of QR factorization is to the solution of least-squares problems (Björck (1996)). The techniques used here carry over from matrices to quasimatrices with virtually no changes. Given a quasimatrix $A$ of full rank and a function $f$, both defined on $[a, b]$, we seek a vector $c$ such that

$$
\|A c-f\|=\text { minimum. }
$$

As first worked out fully in Golub (1965), the solution can be obtained by solving the triangular system of equations

$$
R c=Q^{*} f,
$$

with $Q$ and $R$ defined by (2.1). For this computation it is not necessary to form $Q$; it is enough if the Householder vectors $v_{k}$ are stored and then used to compute $Q^{*} f$. One can also write (3.2) as $c=A^{+} f$, where $A^{+}$is the pseudoinverse of $A$, defined by

$$
A^{+}=R^{-1} Q^{*} .
$$

The pseudoinverse is an $n \times \infty$ quasimatrix: it has $n$ "rows," each of which is a function on $[a, b]$.

\section{Singular value decomposition and related computations}

Once the $\mathrm{QR}$ factorization (2.1) of a quasimatrix $A$ is known, it is an easy matter to compute its singular value decomposition $A=U \Sigma V^{*}$. Following Battles \& Trefethen (2004), we compute first the singular value decomposition $U_{1} \Sigma V^{*}$ of $R$, a standard problem since $R$ is an ordinary matrix. We then set $U=$ $Q U_{1}$, giving

$$
A=\left(Q U_{1}\right) \Sigma V^{*}=U \Sigma V^{*} .
$$

Here $U$ is $\infty \times n, \Sigma$ is $n \times n$ with diagonal form and nonincreasing nonnegative diagonal entries $\left\{\sigma_{j}\right\}$, and $V$ is $n \times n$ and unitary. This also gives us an alternative and numerically more stable formula for the pseudoinverse:

$$
A^{+}=V \Sigma^{-1} U^{*} .
$$

As in the case of QR factorization, an alternative but less stable way to compute the SVD would be to compute $A^{*} A$ and then work from the SVD of this $n \times n$ matrix.

From the SVD of $A$ we can extract its norm,

$$
\|A\|=\sigma_{1},
$$

and its condition number,

$$
\kappa(A)=\sigma_{1} / \sigma_{n},
$$

where $\sigma_{1}$ and $\sigma_{n}$ denote the largest and smallest singular values, respectively. Geometrically, these numbers have their usual interpretations as the lengths of the largest and smallest semiaxes of the $n$ dimensional hyperellipsoid that is the image under $A$ of the unit ball in $\mathbb{C}^{n}$; the only difference from the matrix case is that this hyperellipsoid, while still itself finite-dimensional, is now a subset of the infinite-dimensional space $L_{2}[a, b]$. If some of the singular values are zero, then $A$ is rank-deficient, and its rank can be determined by counting the nonzero singular values. For a numerical rank determination algorithm one introduces a tolerance defining an approximate notion of "nonzero". 
The method of computation of the SVD summarized in (4.1), making use of a preliminary QR factorization, is in the matrix case sometimes known as the "Lawson-Hanson-Chan SVD" (Chan (1982); Lawson \& Hanson (1974); Trefethen \& Bau (1997)). For $m \times n$ matrices it is recommended in cases with $m>5 n / 3$, so it seems natural that it should be appropriate in the quasimatrix case " $m=\infty$ ". On the other hand it is also possible to devise a quasimatrix SVD algorithm of the "Golub-Kahan" variety, in which one proceeds directly to bidiagonal form without a preliminary QR factorization (Golub \& Kahan (1965)). This would involve $n$ quasimatrix Householder reflections on the left, as we have used already, interleaved with $n-1$ matrix Householder reflections on the right. If $G^{*}$ denotes the product of these matrix Householder reflections, then the form that results from Golub-Kahan bidiagonalization is

$$
H A=E B G^{*},
$$

where $B$ is an $n \times n$ upper-bidiagonal matrix and $G$ is $n n \times n$ unitary matrix of dimensions $n \times n$. If $U_{1} \Sigma V_{1}^{*}$ is an SVD of $B$, then the SVD of $A$ can be written

$$
A=\left(H^{*} E U_{1}\right) \Sigma\left(G V_{1}\right)^{*} \text {. }
$$

\section{Further algorithmic details}

Operation counts. For matrix QR decomposition, the Gram-Schmidt algorithm requires $\sim n^{2} / 2$ inner products and $\sim n^{2} / 2$ saxpys (scalar times vector plus vector). For $m \gg n$, this corresponds to a total operation count of $\sim 2 m n^{2}$ flops, where a flop is an addition, subtraction, multiplication or division. Householder triangularization costs the same if you only need $R$, but if $Q$ must be formed as well, the operation counts double to $\sim n^{2}$ inner products and $\sim n^{2}$ saxpys.

In the quasimatrix case, the Gram-Schmidt numbers are again $\sim n^{2} / 2$ inner products and $\sim n^{2} / 2$ saxpys (scalar times vector plus vector). Of course, in this context the meaning of an inner product or a saxpy is different, since functions and quadrature are involved rather than discrete vectors. As for Householder triangularization of a quasimatrix, the second phase of forming $Q$ again requires $\sim n^{2} / 2$ inner products and $\sim n^{2} / 2$ saxpys. The triangularization, however, is three times as expensive as before, at least with the extra line to "improve orthogonality," requiring $\sim 3 n^{2} / 2$ inner products and $\sim 3 n^{2} / 2$ saxpys. Thus roughly speaking, whereas Householder QR factorization is twice as expensive than Gram-Schmidt for matrices, it is three or four times as expensive for quasimatrices. The reason are the extra orthogonalization operations needed at two points in the algorithm, which for matrices are bypassed since they are implied by the sparsity structure. We suspect it may be possible to improve these figures.

Sparsity and Givens rotations. For matrix QR factorization, an alternative to Householder reflections is Givens rotations, which act on two rows at a time rather than $O(m)$ rows. Though more expensive when the matrix is dense, this technique may have advantages when the matrix is sparse. Now, what might it mean to say that a quasimatrix $A$ is sparse? A possible answer would be that each column of $A$ is orthogonal to most columns of $E$, assuming that $E$ is specified in advance. Perhaps there may be problems where this property arises and analogues of Givens operations would be useful, but we have not pursued this idea.

\section{Chebfun examples}

This work was motivated by the chebfun software system, which makes it possible to compute with quasimatrices in MATLAB (Driscoll et al. (2008)). Chebfun Version 1 used Gram-Schmidt orthogonalization, but beginning with Chebfun Version 2.0308 in July 2008, we have replaced Gram-Schmidt 
orthogonalization by Householder triangularization in all QR factorizations. We close with a few examples involving QR factorization and SVD computations in this system.

First, here is a computation of the norm and condition number of the set of functions $1, x, x^{2}, \ldots, x^{5}$ over $[-1,1]$ :

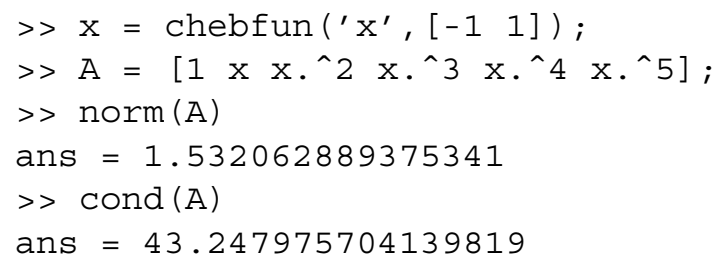

If $[-1,1]$ is changed to $[0,1]$, the norm decreases to 1.272359956507724 and the condition number increases to 3866.659881620226 .

Similarly, suppose we want to know the dimension of the space spanned by the functions $1, \sin ^{2}(x)$, and $\cos ^{2}(x)$. The following result comes out the same with either of the choices of domain of $x$ mentioned above:

$>\operatorname{rank}\left(\left[1 \sin (x) \cdot{ }^{\wedge} 2 \cos (x) \cdot \wedge 2\right]\right)$

$>$ ans $=2$

Here is an example involving piecewise smooth chebfuns. The commands

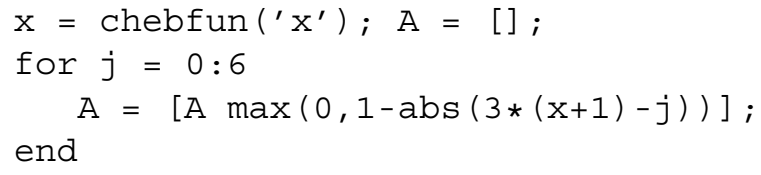

construct an $\infty \times 7$ quasimatrix on $[-1,1]$ whose columns correspond to triangular hat functions of width $1 / 3$ centered at $-1,-2 / 3,-1 / 3, \ldots, 1$. Linear combinations of these functions are piecewise linear functions on $[-1,1]$ with breakpoints at $-2 / 3,-1 / 3, \ldots, 2 / 3$. The following code computes the least-squares fit by such piecewise linear functions to $f(x)=e^{x} \sin (6 x)$ and produces the plot shown in Figure 1:

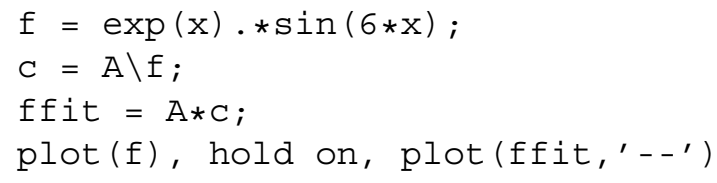

Here is the norm of the residual:

$>\operatorname{norm}(\mathrm{f}-\mathrm{ffit})$

ans $=0.301000501411522$ 


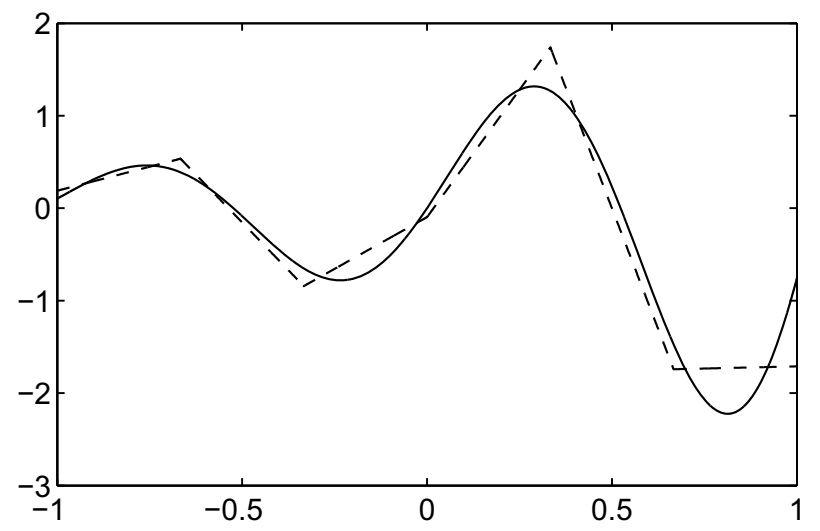

FIG. 1. Quasimatrix least-squares fit by piecewise linear functions computed with the " $\backslash$ " command in the chebfun system. The residual norm minimized is defined by integrals, not point values. This quasimatrix has condition number 1.974212678743394 .

All these computations are continuous, involving integrals, not just point values.

Finally, we recall that the advantage of Householder triangularization over Gram-Schmidt orthogonalization is that it delivers an orthonormal quasimatrix $Q$, even when $A$ is rank-deficient. We can verify this numerically by considering the highly rank-deficient quasimatrix consisting of two copies of $A$ placed beside each other:

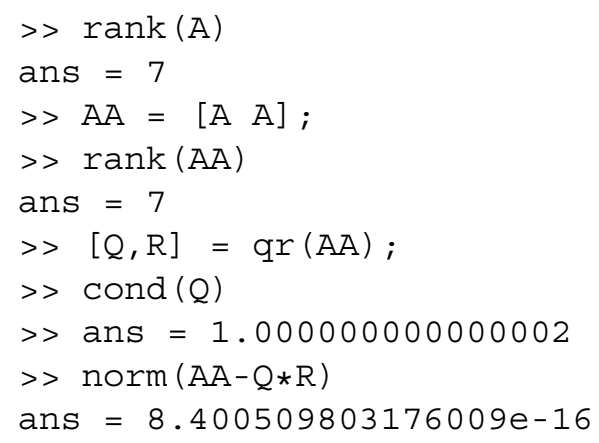

The chebfun system is freely available, together with users guides and sample codes and other materials, at http://www.comlab.ox.ac.uk/chebfun.

\section{Discussion}

What is the essential algorithmic difference between factorizations of matrices and quasimatrices? Perhaps the main answer has to do with the notion of zero entries and associated matters of structures and sparsity. With ordinary matrices, the problem has already been formulated in the basis $e_{1}, e_{2}, \ldots$ : to find out if a vector has zero component in a direction $e_{j}$, we need only check if a certain number is zero. With quasimatrices, there is no basis given a priori. We must choose one to work with in practice- here we have taken suitably scaled Legendre polynomials - but the check of whether a function has zero component in a direction $e_{j}$ now requires the computation of an inner product. 
This note is a contribution to a larger project: to reconstruct the subject of numerical linear algebra in the context of functions rather than vectors. The project has an intellectual side, as it forces us to confront fundamental questions of the deeper meanings of the algorithms we know and trust so well; and it has a practical side, a long-term enterprise indeed, which is to enable computational science and engineering to work directly with functions, with details of discretizations hidden away just as details of floating point arithmetic are today hidden away when we work with numbers.

\section{Acknowledgments}

This article was mostly written during the 17th Householder Symposium, held in June, 2008 in Zeuthen, Germany. I am grateful for advice from several of the participants at the meeting and others including Nick Higham, Sasha Malyshev, Chris Paige, Beresford Parlett, Pete Stewart, and Gil Strang. My chebfun teammates Toby Driscoll, Ricardo Pachón, and Rodrigo Platte have also been very helpful. This work was funded by EPSRC grant EP/E045847/1.

\section{REFERENCES}

Battles, Z. (2006) Numerical Linear Algebra for Continuous Functions, DPhil thesis, Oxford University Computing Laboratory.

Battles, Z. \& TREFEthen, L. N. (2004) An extension of MATLAB to continuous functions and operators, SIAM J. Sci. Comp., 25, 1743-1770.

BJÖRCK, Å (1996) Numerical Methods for Least Squares Problems. Philadelphia: SIAM.

CHAN, T. F. (1982) An improved algorithm for computing the singular value decomposition, ACM Trans. Math. Softw., 8, 72-83.

DE BOOR,C. (1991) An alternative approach to (the teaching of) rank, basis, and dimension, Lin. Alg. Appl., 146, 221-229.

Driscoll, T. A., Pachón, R., Platte, R. \& Trefethen, L. N., Chebfun Version 2, www. comlab.ox.ac.uk/chebfun/.

Golub, G. H. (1965) Numerical methods for solving least squares problems, Numer. Math., 7, 206-216.

Golub, G. H. \& KAHAN, W. (1965) Calculating the singular values and pseudoinverse of a matrix, SIAM J. Numer. Anal., 2, 205-224.

Golub, G. H. \& VAn LoAn, C. (1996) Matrix Computations, 3rd ed. Baltimore: Johns Hopkins University Press.

Higham, N. J. (2002) Accuracy and Stability of Numerical Algorithms, 2nd ed. Philadelphia: SIAM.

HouseHolder, A. S. (1958) Unitary triangularization of a nonsymmetric matrix, J. Assoc. Comput. Mach., 5, 339-342.

Lawson, C. L. And Hanson, R. J. (1974) Solving Least Squares Problems. Englewood Cliffs, NJ: Prentice Hall.

Stewart, G. W. (1998) Afternotes Goes to Graduate School. Philadelphia: SIAM.

Stewart, G. W. (1998b) Matrix Algorithms, v. 1: Basic Decompositions. Philadelphia: SIAM.

L. N. Trefethen And D. BAU, III (1997) Numerical Linear Algebra. Philadelphia: SIAM. 